\title{
ACTIVE FREQUENCY TUNING FOR MICRORESONATORS BY LOCALIZED THERMAL STRESSING EFFECTS
}

\author{
TODD REMTEMA ${ }^{1}$ and LIWEI LIN \\ Department of Mechanical Engineering University of California, Berkeley \\ 5126 Etcheverry Hall, MC 1740, CA 94720-1740 \\ TEL: 510-643-5495, FAX: 510-643-5599, lwlin@me.berkeley.edu
}

\begin{abstract}
Active frequency tuning for comb-shape micro resonators has been successfully demonstrated by means of localized stressing effects. A mechanical beam structure that can be resistively heated to generate thermal stress is integrated as part of the micro resonator for frequency tuning. Experimentally, frequency change up to $6.5 \%$ is measured for resonators with central frequency around $31 \mathrm{kHz}$. The required tuning power is $25 \mathrm{~mW}$ in the form of localized joule heating. Analytically, both a one-dimensional electrothermal model and a dynamic model are established to characterize electrical, thermal and frequency responses of active frequency tuning. The simulation results of frequency spectrum are consistent with experimental measurements. A reliability test has been conducted for more than 300 million cycles under $6.5 \%$ of frequency tuning range and reveds no malerial damages on the micro resonator. This scheme enables active frequency tuning under low power consumption, independent of the input/output function of a micro resonator. As such, it has potential applications to resonator based MEMS devices, such as rate gyroscopes and microelectromechanical filters.
\end{abstract}

\section{INTRODUCTION}

Micro resonators have been used in various resonator-based microsystems, such as resonant accelerometers [1,2], microelectromechanical filters [3-5], and gyroscopes [6]. One common roadblock in the manufacturing process comes from local process variations. As a result, even though the micro fabrication process is well-controlled, structural deviations exist between designed and fabricated devices and between same devices adjacent to each other on the same wafer. Therefore, it is common that the frequency responses of micro resonators may be off from the desired design value and should be eradicated by post-tuning processes. For example, up to several percentage variations of resonant frequency can be found in the widely used comb-shape micro resonators on the same chip [7].

Frequency tuning methods can be split into two major categories, those that make one time permanent changes and those that make active adjustments without permanent damages to microstructures. The permanent changes can be accomplished by annealing the folded springs of a comb structure using voltage pulses to change the stiffness and quality factor [8] or by a post-deposition process [9]. On the active frequency tuning side, three basic methods have been investigated. The first of these is tuning via an "electrostatic stiffness" change, where the stiffness can be adjusted electrostatically with capacitive structures [6,10-13]. The second method tunes by making changes in the internal stresses of the structure to induce changes in resonance $[14,15]$. The third method takes advantage of the inherent temperature dependence of Young's modulus [16]. The method presented in this work takes advantage of the temperature dependence of Young's modulus exhibited by most materials as well as the thermally induced internal stress effects. That is, as the temperature of polysilicon goes up its Young's modulus drops and this will cause a minor reduction in the resonant frequency. The localized thermal stress generated by joule heating plays a major role in the proposed frequency tuning scheme. If a compressive stress is generated, the structure becomes more compliant resulting in a lower resonant frequency. If a tensile stress is generated, the structure becomes stiffer resulting in a higher resonant frequency. Through the use of fllament like heating as implemented in this work, the device heats up resulting in a decrease in Young's modulus and an increase in compressive stresses. Both effects help to actively lower the resonant frequency.

\section{A TUNABLE MICRO RESONATOR}

A tunable micro resonator by means of localized thermal stressing effects has been designed as illustrated in Fig. 1. The comb drive structure is modified by replacing one folded-beam suspension with a straight suspension as the tuning beam. The folded beam suspension helps minimizing the non-linear effect that may arise from the straight-beam structure design $[17,18]$. One drawback of this tolded-beam/straight-beam design as opposed to a straightbeam/straight-beam design is that the tuning range of the device is reduced. However, most non-linear effects are avoided. The straight-beam suspension is $4 \mu \mathrm{m}$ wide and $165 \mu \mathrm{m}$ long. On the folded-beam suspension, the four beams are $2 \mu \mathrm{m}$ wide and $150 \mu \mathrm{m}$ long and the truss is $5 \mu \mathrm{m}$ wide and $36 \mu \mathrm{m}$ long. The anchors on the two sides of the suspension system are electrically isolated such that a voltage can be applied across the structure. This voltage heats the structure through filament like heating that causes the structure itself to heat up and to expand. During the heating process the substrate stays relatively cool due to the rapid heat transfer capabilities of silicon.

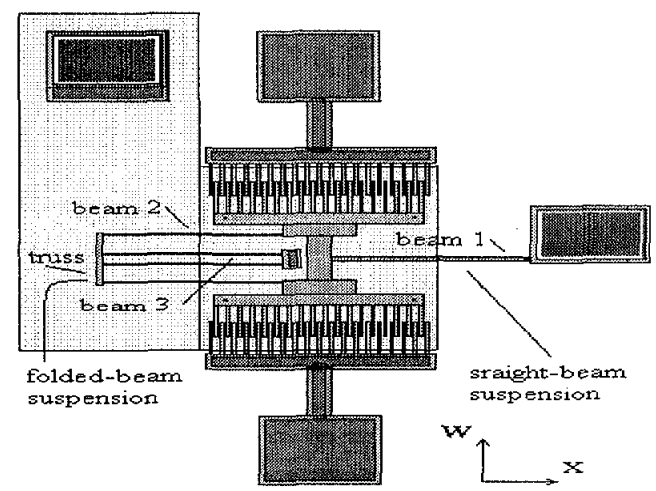

Fig. 1, Schematic diagram of a comb-shape micro resonator with a straight-beam for active frequency tuning via localized stressing effects.

\footnotetext{
${ }^{1}$ Mechanical Engineering and Applied Mechanics Department, the University of Michigan
} 
A standard surface-micromachining process provided by MCNC [19] is used to fabricate these tunable micro resonators and all the simulation and experimental measurements presented here are based on the MUMPS-22 run. A sheet resistance of $6.4 \mathrm{~s} / \mathrm{square}$ and a thermal coefficient of resistivity of $1.7 \times 10^{-3} \mathrm{ohm} /{ }^{\circ} \mathrm{K}$ are measured for the structural polysilicon layer. Young's modulus and density of the polysilicon layer used in this work are $160 \mathrm{GPa}$ and $2300 \mathrm{~kg} / \mathrm{m}^{3}$. The thermal coefficient of expansion and specific heat of polysilicon are adopted from silicon as $2.6 \times 10^{-6} \% \mathrm{~K}$ and 712 $\mathrm{J} /\left(\mathrm{kg}^{\circ} \mathrm{K}\right)$, respectively. The values of thermal conductivity of the silicon, polysilicon, and nitride layers used in this paper are 148, 34, and $1.6 \mathrm{~W} /\left(\mathrm{m}^{\circ} \mathrm{K}\right)$.

The frequency responses of the device are measured using a CCD camera built into a probe station. Pictures are first taken and then opened in Canvas ${ }^{\mathrm{TM}}[201$ and the amplitudes of vibration for every $100 \mathrm{~Hz}$ of driving frequency are measured and recorded. Measurements of resonance are obtained by adjusting the driving frequency until the peak driving amplitude of the device could be observed. This method could locate the resonant frequency within $100 \mathrm{~Hz}$ when the resonant frequency is typically around $30 \mathrm{kHz}$. This gives an accuracy of roughly $0.33 \%$.

\section{EXPERIMENTAL RESULTS AND DISCUSIONS}

A dynamic model and a one-dimensional electro-thermal model have been established [21]. With this model, the transient responses of the temperature versus time can be graphed as seen in Fig. 2. The rising and falling times for the system to reach steady state under heating and cooling are found to be around $140 \mu \mathrm{sec}$. Using the model developed above, a temperature profile of the system at a time significantly larger than the rise time can be attained as shown in Fig. 3. Nodes 1 to 25 are the inner portion of the foldedbeam suspension, nodes 26 to 32 are the truss portion of the foldedbeam suspension, nodes 33 to 58 are the outer portion of the foldedbeam suspension, node 59 is the rigid central mass, and nodes 60 to 100 are the straight-beam suspension.

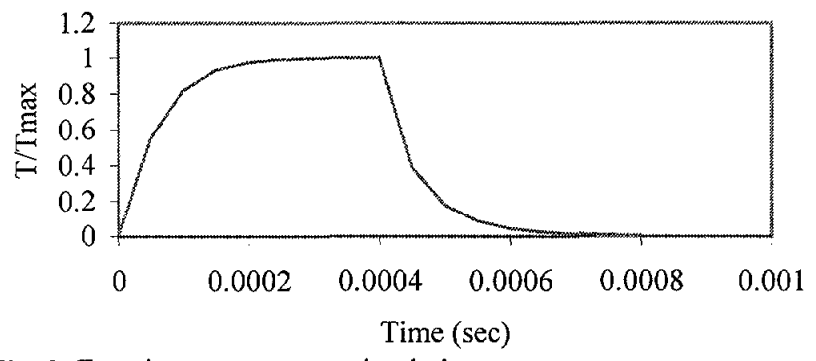

Fig. 2, Transient temperature simulation.

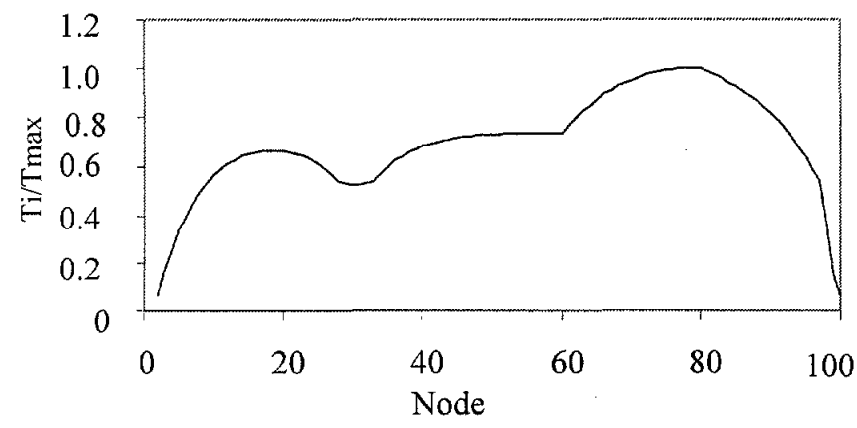

Fig. 3, Temperature profile simulated by the electro-thermal model.
Figure 4 is an optical photo showing the tunable micro resonator under a microscope. When a high tuning power is supplied for resistive heating of the system and the background light is turned off, Fig. 5 shows the hot spots. When Fig. 5 is compared with Fig. 3, the highest temperature region agrees well with what is seen experimentally. The temperature simulations under various input power are used to predict the thermal expansion of comb-drive to derive the tuning force $\mathrm{P}$ and frequency changes.

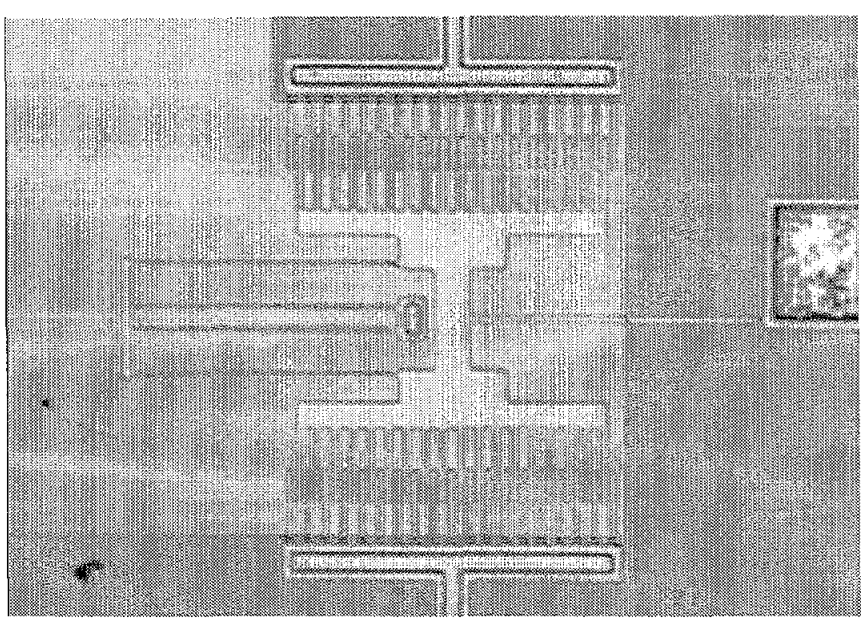

Fig. 4, A tunable resonator under an optical microscope.

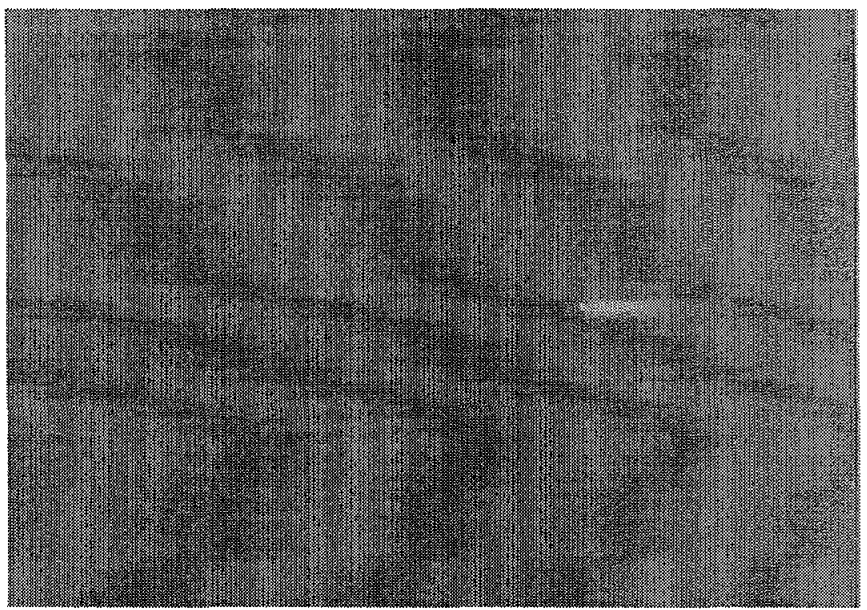

Fig. 5, Hot spot is shown under the joule heating effect when the illumination of the optical microscope is turned off.

Using the experimental setup described earlier, four basic tests have been conducted on the tunable resonator. The first test is a comparative test where the resonator is heated by external means and by localized joule heating, respectively. The external heating is accomplished by placing the device on a hot plate. In both tests, the resistance of the suspended beams is measured at various temperatures when resonant frequency change is measured. The resistance is used as the guidance to estimate the temperature of the device. Figure 6 shows the resonant frequency changes with respect to the resistance changes (tempcrature changes). It is found that when the resistance is at $940 \mathrm{ohm}$ it corresponds to an average temperature of $150^{\circ} \mathrm{C}$. At this temperature, the external heating causes a frequency change of about $1 \%$ and the internal joule heating makes the frequency change of $6.5 \%$. Under this joule heating condition, the maximum temperature at the tuning beam is $255^{\circ} \mathrm{C}$ and the average temperature of the resonator is $150^{\circ} \mathrm{C}$. 


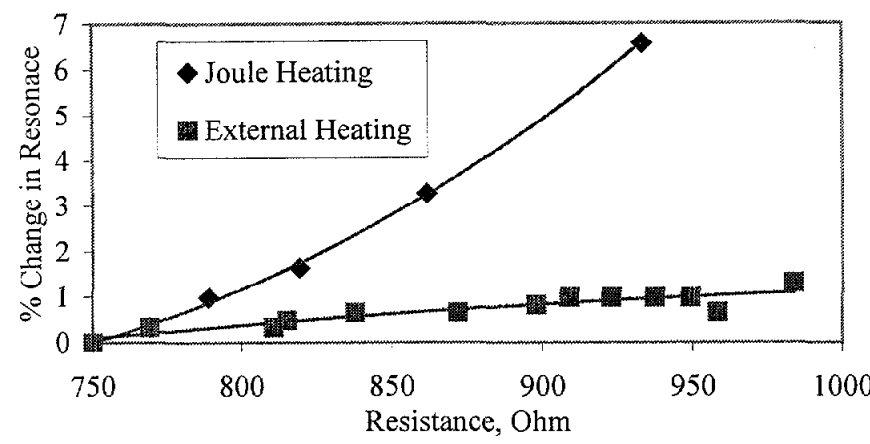

Fig. 6, Frequency changes by means of external (global) heating and joule (localized) heating.

It is concluded that under the same average temperature, the joule heating causes the resonance to change much more than the external heating. The external heating makes both the substrate and the device to expand such that this keeps the thermal stressing effects to a minimum. The change in resonance by external heating can be attributed mainly to the change in Young's modulus and partially to the change in density and thermal mismatch. The temperature coefficient of Young's modulus can be found using the following equation [3]:

$$
\mathrm{TC}_{f}=\frac{1}{2}\left(\mathrm{TC}_{\mathrm{E}}-\mathrm{TC}_{\mathrm{b}}\right)
$$

Where $T C_{f}$ is the temperature coefficient of the resonant frequency, $\mathrm{TC}_{\mathrm{E}}$ is the temperature coefficient of Young's modulus and $\mathrm{TC}_{\mathrm{h}}$ is the temperature coefficient of thermal expansion. In this work, $\mathrm{TC}_{\mathrm{E}}$ is found to be $-40 \mathrm{ppm} /{ }^{\circ} \mathrm{C}$ by the external heating experiments, which is close to previously reported values [3-5]. In the joule heating case the device itself heats up where as the substrate stays relatively cool. This creates a large internal stressing effect as well as a change in Young's modulus.

The second experiment is to check for repeatability and compliance of the dynamic and electrothermal models. In this test, five different structures from four different dice are tested over a range of tuning powers. The change in resonance versus the applied tuning power is plotted for 5 different devices along with the predicted theoretical response as shown in Fig. 7. As seen in the plot, the theory predicts a quasi-linear change in resonance versus input power. The experimental data points are close to the theoretical prediction when the input power is lower than $30 \mathrm{~mW}$. However, the model is only valid for a first-order approximation because it does not take into account several secondary effects, including non-linearity due to the softening of the spring as the structure deflects, the change in specific heat, thermal conductivity, and the thermal coefficient of expansion as the temperature increases.

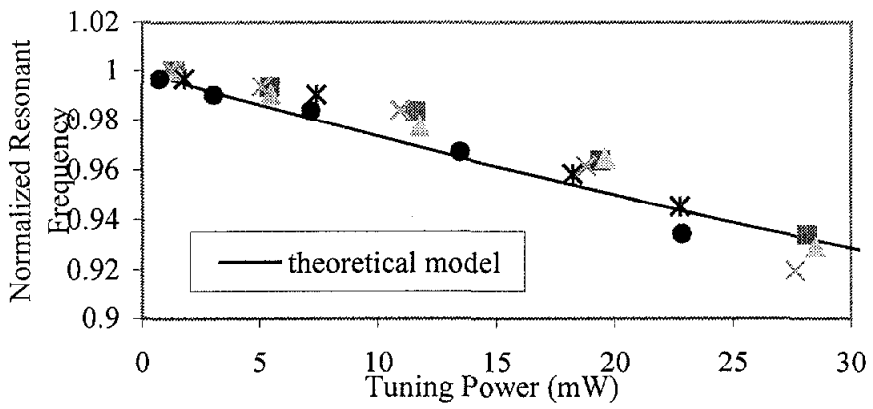

Fig. 7, Measured frequency changes vs. tuning power for 5 different devices compared to the theoretical model.
The third test is to measure the spectrum response of the resonator. One device is tested in this manner. The driving frequency is changed every $100 \mathrm{~Hz}$ and the amplitude is recorded. This is done by scanning both into the higher frequencies and into the lower frequencies until the amplitude is relatively low. Several tuning currents are conducted and recorded as shown in Fig. 8 from 0 to $5 \mathrm{~mA}$. As seen in the plot, the system starts out with a fairly linear response. As the tuning current is increased the response begins to go more and more non-linear. This effect is to be expected. Non-linearity arises from the stiffening of the straightbeam suspension as the device deflects. The stiffening comes from the stretching of the beam as it deflects. This stretching increases the restoring force of the spring. It is well documented that as the magnitude of the intrinsic stress goes up the non-linearity also goes up [12]. Also seen in the plot is that the quality factor of the system stays nearly the same at about 50 . This is because most of the dampening comes from the friction with the air that remains relatively unchanged during the frequency tuning process.

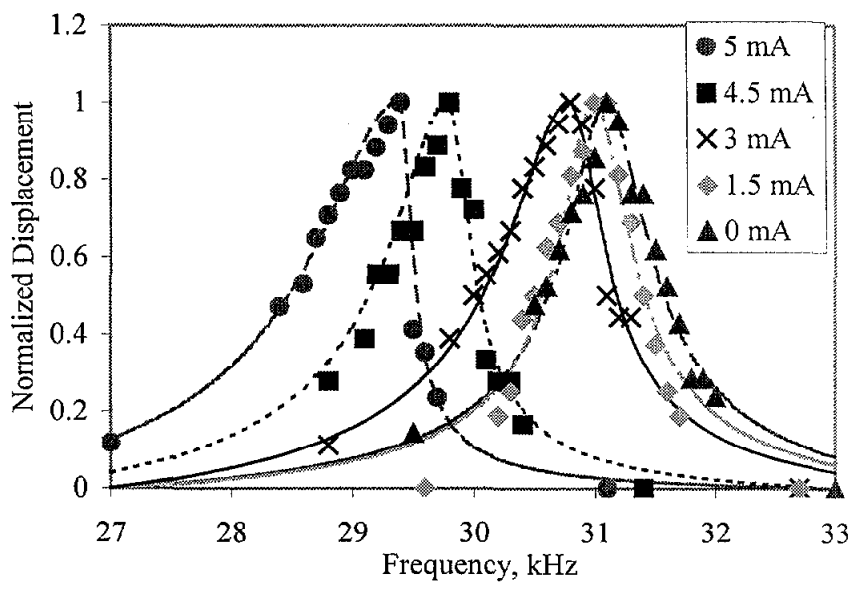

Fig. 8, Frequency spectrum of one device at 5 different tuning amperages from 0 to $5 \mathrm{~mA}$.

The final test is the reliability experiment for the tuning method. A resonator is tuned to $1 \%$ and again to $6.5 \%$ for approximate 300 million cycles, respectively. At the end of these tests, no visual damages can be found on the device. Most importantly, it returns completely to its original natural frequency and the measured resistance does not change before and after the tests. This indicates that there is no structural degradation and the active tuning method via thermal stressing effects as presented is feasible for repeated, long-term applications. Although structural damage is not expected at the tuning power of $6.5 \%$ with a highest temperature at $255^{\circ} \mathrm{C}$, further investigations should be conducted for the fatigue and creep [22] phenomena under high temperature and high stress operations.

\section{CONCLUSION}

An active frequency tuning mechanism has been proposed and has been proven to adjust the natural frequency of comb-shape micro resonators up to $6.5 \%$ with minimal power requirements at $25 \mathrm{~mW}$. This method can be applied at the component level after the completion of the packaging process and it can actively tune the frequency of micro resonators with a response time of $140 \mu \mathrm{sec}$. It is important for microstructures to have frequency tuning capabilities in order to adjust for manufacturing variations in microsystems. Both dynamic and electrothermal models are established in this work to provide the design guidelines for active frequency tuning. Four 
different kinds of experiments have been conducted. The experiment of external versus joule heating demonstrates that the thermal stressing effect is the dominant factor in the proposed tuning mechanism. The second experiment measured 5 different devices for frequency changes at various tuning powers. It is concluded that the analytical model is consistent with the expcrimental results. The third experiment measured the spectrum of comb-resonator at various tuning power. It is found that nonlinear effects begin at high tuning power probably due to the spring stiffening effect. Finally, long-term tests for 300 million cycles demonstrate the good reliability of the proposed tuning mechanism.

\section{ACKNOWLEDGEMENT}

The authors would like to thank Mr. M. Chiao for discussions about the electrothermal model, Mr. J.-H. Tsai and Mr. B. Casey for wire bonding and packaging of the resonators. This work is supported in part by an NSF CAREER award (ECS-0096098) and a DARPA/MTO/MEMS grant (F30602-98-2-0227).

\section{REFERENCES}

1. T.A. Roessig, R.T. Howe, A.P. Pisano and J.H. Smith, "Surfacemicromachined resonant accelerometer," Intl. Conf. On SolidState Sensors and Actuators (Transducers 97), Chicago, 1997, pp. 859-862.

2. Y. Omura, Y. Nonomura and O. Tabata, "New resonant accelerometer based on rigidity change," Intl. Conf. On SolidState Sensors and Actuators (Transducers 97), Chicago, 1997, pp. 855-858.

3. L. Lin, R.T. Howe and A.P. Pisano, "Microelectromechanical filters for signal processing," Journal of Microelectromechanical systems. Vol. 7. No. 3. September 1998, pp. 286-294.

4. K. Wang and C. T.-C. Nguyen, "High-order micromechanical electronic filters," Proceedings of 1997 IEEE International Micro Electro Mechanical Systems Workshop, Nagoya, Japan, 1997, pp. 25-30.

5. C. T.-C. Nguyen, "Frequency-selective MEMS for miniaturized low-power communication devices," IEEE Transactions on Microwave Theory and Techniques, Vol. 47, No. 8, pp. 14861503.

6. Y. OH, B. Lee, S. Baek, H. Kim, J. Kim, S. Kang and C. Song, "Surface-micromachined tunable vibratory gyroscope," Proceedings, 1997 IEEE International Micro Electro Mechanical Systems Workshop, Nagoya, Japan, 1997, pp. 272-277.

7. W. C. Tang, T.C. H. Nguyen and R. T. Howe, "Laterally driven polysilicon resonant microstructures," Sensors and Actuators, Vol.20, 1989. pp.25-32.

8. K. Wang, A.-C. Wong, W.-T. Hsu and C. T.-C. Nguyen, "Frequency trimming and Q-factor enhancement of micromechanical resonators via localized filament anealing," Intl. Conf. On Solid-State Sensors and Actuators (Transducers 97), Chicago, 1997, pp. 109-112.

9. D. Joachim and L. Lin, "Localized deposition of polysilicon for MEMS post-fabrication processing," 1999 ASME International Mechanical Engineering Congress and Exposition, Proceedings of Microelectromechanical Systems, MEMS-Vol. 1, pp. 37-42, Nashville, Tennessee, 1999.

10. K. Lee and Y. Cho, "Frequency tuning of laterally driven microresonator using an electrostatic comb array of linearly varied length," Intl. Conf. On Solid-State Sensors and Actuators (Transducers 97), Chicago, 1997, pp. 113-116.
11. S.G. Adams, F.M. Bertsch, K.A. Shaw, P.G. Hartwell, F.C. Moon and N.C. Macdonald, "Capacitance based tunable resonators," J. micromech. Microeng. Vol. 8, 1998, pp. 15-23.

12. C. Gui, R. Legtenberg, H. Tilmans, J. Fluitman and $M$. Elwenspoek, "Nonlinearity and hysteresis of resonant strain gauges," Journal of Microelectromechanical systems. Vol. 7. No. 1. March 1998, pp. 122-127.

13. S. G. Adams, F. M. Bertsch, K. A. Shaw and N. C. MacDonald "Independent tuning of lineat and nonlinear stiffness coefficients," Journal of Microelectromechanical systems. Vol. 7. No. 2. June 1998, pp. 172-180.

14. J.J. Yao and N.C. MacDonald, "A micromachined, single-crystal, silicon, tunable resonator," J. micromech. Microeng. Vol. 6, 1996, pp. 257-264.

15. R. R. A. Syms, "Electrothermal frequency tuning of folded and coupled vibrating micromechanical resonators," Journal of Microelectromechanical systems. Vol. 7. No. 2. June 1998, pp. 164-171.

16. H. Kahn, M.A. Huff and A.H. Heuer, "Heating effects on the Young's modulus of films sputtered onto micromachined resonators," Mat. Res. Soc. Proc. Vol. 518, 1998, pp. 33-38.

17. R.I. Pratt, G.C. Johnson, R.T. Howe and J.C. Chang, "Mechanical structures for thin film characterization," in Dig. Transducers '91, Int. Conf. Solid-State Sensors and Actuators, pp. 205-208.

18. H. Tilmans, M. Elwenspoek and J. Fluitman, "Micro resonant force gauges," Sensors and Actuators, Vol. A30, 1992, pp. 3553.

19. MCNC, MEMS Technology Applications Center, Research Triangle Park, NC, 27709.

20. Canvas. Graphics Software. Deneba Software, Inc., 7400 S.W. 87th Avenue, Miami, Florida 33173, USA, 1986.

21. Todd Remthma, "Active Frequency Tuning Via Localized Thermal Stressing Effects," Master Thesis, University of Michigan, 1999.

22. K.S. Teh, Liwei Lin and M. Chiao, "The creep behaviour of polysilicon microstructure," 10th Int. Conference on Solid State Sensors and Actuators, Transducer's 99, Technical Digest, Sendai, Japan, June 1999, pp. 508-511. 